\title{
Spatio-temporal chaos in duopoly games
}

\author{
Risong Li, ${ }^{\mathrm{a}, *}$, Yu Zhao ${ }^{\mathrm{a}}$, Tianxiu Lu ${ }^{\mathrm{b}, \mathrm{c}}, \mathrm{Ru}$ Jiang $^{\mathrm{a}}$, Hongqing Wang ${ }^{\mathrm{a}}$, Haihua Liang ${ }^{\mathrm{a}}$ \\ ${ }^{a}$ School of Mathematic and Computer Science, Guangdong Ocean University, Zhanjiang, Guangdong, 524025, P. R. China. \\ ${ }^{b}$ Department of Mathematics, Sichuan University of Science and Engineering, Zigong, Sichuan, 643000, P. R. China. \\ ${ }^{c}$ Artificial Intelligence Key Laboratory of Sichuan Province, Zigong, Sichuan, 643000, P. R. China.
}

Communicated by A. Atangana

\begin{abstract}
Suppose that $G$ and $H$ are two given closed subintervals of $\mathbb{R}$, and that $q: G \rightarrow H$ and $p: H \rightarrow G$ are continuous maps. Let $\Gamma(s, t)=(p(t), q(s))$ be a Cournot map over the space $\mathrm{G} \times \mathrm{H}$. In this paper, we study spatio-temporal chaos of such a Cournot map. In particular, it is shown that if $p$ and $q$ are onto maps, then the following are equivalent:

1) $\Gamma$ is spatio-temporally chaotic;

2) $\left.\Gamma^{2}\right|_{\Lambda_{1}}$ is spatio-temporally chaotic;

3) $\left.\Gamma^{2}\right|_{\Lambda_{2}}$ is spatio-temporally chaotic;

4) $\left.\Gamma\right|_{\Lambda_{1} \cup \Lambda_{2}}$ is spatio-temporally chaotic.

Moreover, it is proved that if $p$ and $q$ are onto maps, then $p \circ q$ is spatio-temporally chaotic if and only if so is $q \circ p$. Also, we give two examples which show that for the above results, it is necessary to assume that $p$ and $q$ are onto maps. (C) 2017 All rights reserved.
\end{abstract}

Keywords: Spatio-temporal chaos, Li-Yorke sensitivity, duopoly game.

2010 MSC: 37D45, 54H20, 37B40, 26A18, $28 \mathrm{D} 20$.

\section{Introduction}

Let $G$ and $H$ be closed subintervals of $\mathbb{R}$, and let $p: H \rightarrow G$ and $q: G \rightarrow H$ be continuous. In the whole paper, we always let $\Gamma: G \times G \rightarrow G \times H$ be defined as $\Gamma(s, t)=(p(t), q(s))$ for any $(s, t) \in G \times H$. Such a map has been investigated to give a mathematical analysis of Cournot duopoly (see [17]). Probably the first notion of chaos in a mathematically rigorous way was posed by Li and Yorke [12]. Since then, a lot of different notions of chaos have been posed. Akin and Kolyada gave the concept of Li-Yorke sensitivity for the first time and the concept of spatio-temporal chaos [1]. Schweizer and Smítal [20] gave the concept of distributional chaos. We know that distributional chaos is equivalent to positive topological entropy and some other chaotic properties for some particular spaces (see [14, 20,31]), and that this equivalence relationship will become invalid for some higher dimensional spaces [21] and some zero-dimensional

\footnotetext{
${ }^{*}$ Corresponding author

Email addresses: gdoulrs@163.com (Risong Li), datom@189.cn (Yu Zhao), lubeeltx@163.com (Tianxiu Lu), jiru1995@163.com (Ru Jiang), wanghq3333@126.com (Hongqing Wang), lhhlucy@126.com (Haihua Liang)

doi:10.22436/jnsa.010.07.33
} 
spaces [15]. Wang et al. [22] gave the definition of distributional chaos with respect to a sequence and got that this kind of chaos is equivalent to Li-Yorke chaos for continuous maps over a closed subinterval. For many related notions and dynamics from dynamical system, we refer the reader to [10, 23, 25, 26, 2833]. Over the past few decades, people have been paying very close attention to the chaotic properties of Cournot maps (see $[3-6,13,17,24])$. From $[6,17]$ one can see that there exist Markov perfect equilibria processes. That is, two fixed players move alternatively and ensure that such that any of them chooses the best reply to the previous action of another player. Put $\Lambda_{1}=\{(p(t), t): t \in H\}, \Lambda_{2}=\{(s, q(s)): s \in G\}$, and $\Lambda_{12}=\Lambda_{1} \cup \Lambda_{2}$. Clearly, $\Gamma\left(\Lambda_{12}\right) \subset \Lambda_{12}$. The set $\Lambda_{12}$ is said to be an MPE-set for $\Gamma$ (see [4]). Moreover, in [4] the authors studied several kinds of chaos for Cournot maps, and obtained that for any definition they considered in [4] it does not satisfy the condition that $\Gamma$ is chaotic if and only if so is $\left.\Gamma\right|_{\Lambda_{12}}$. It is well-known that some chaotic properties of Cournot maps have been explored (see $[2,6,8,13,16-18]$ ). Recently, Lu and Zhu further studied some chaotic properties of Cournot maps and showed that some chaotic properties of $\left.\Gamma\right|_{\Lambda_{12}},\left.\Gamma^{2}\right|_{\Lambda_{1}}$, and $\left.\Gamma^{2}\right|_{\Lambda_{2}}$ are same. Then, $\mathrm{Wu}$ [24] proved that $\Lambda$ is Li-Yorke chaotic if and only if $h_{\infty}(p \circ q)=h_{\infty}(q \circ p) \in\{\ln 2,+\infty\}$, where $h_{\infty}(\Lambda)$ is the supremum of all topological sequence entropies of $\Lambda$. In [11], it is proven that for any Cournot map $\Gamma(s, t)=(p(t), q(s))$ over the product space $\mathrm{G} \times \mathrm{H}$, the following properties are satisfied:

(1) if $\Gamma$ is Kato chaotic then so is $\left.\Gamma^{2}\right|_{\Lambda_{1}}$ or $\left.\Gamma^{2}\right|_{\Lambda_{2}}$, where $\Lambda_{1}=\{(p(t), t): t \in H\}$ and $\Lambda_{2}=\{(s, q(s)): s \in G\}$.

(2) it is assumed that $\left.\Gamma^{2}\right|_{\Lambda_{1}}$ and $\left.\Gamma^{2}\right|_{\Lambda_{2}}$ are Kato chaotic, and that the maps $p$ and $q$ satisfy that for any $u>0$, if

$$
\left|(q \circ p)^{n}\left(t_{1}\right)-(q \circ p)^{n}\left(t_{2}\right)\right|<u \quad \text { and } \quad\left|(p \circ q)^{m}\left(s_{1}\right)-(p \circ q)^{m}\left(s_{2}\right)\right|<u
$$

for some integers $n, m>0$, then there is an integer $l(n, m, u)>0$ with

$$
\left|(q \circ p)^{l(n, m, u)}\left(t_{1}\right)-(q \circ p)^{l(n, m, u)}\left(t_{2}\right)\right|<u \text { and } \quad\left|(p \circ q)^{l(n, m, u)}\left(s_{1}\right)-(p \circ q)^{l(n, m, u)}\left(s_{2}\right)\right|<u \text {. }
$$

Then the map $\Gamma$ is Kato chaotic.

In this paper, we continue to explore the spatio-temporally chaotic property of such a Cournot map. In particular, it is proven that if $p$ and $q$ are onto maps, then the following four statements are equivalent:

1) $\Gamma$ is spatio-temporally chaotic;

2) $\Gamma^{2} \mid \Lambda_{1}$ is spatio-temporally chaotic;

3) $\left.\Gamma^{2}\right|_{\Lambda_{2}}$ is spatio-temporally chaotic;

4) $\left.\Gamma\right|_{\Lambda_{1} \cup \Lambda_{2}}$ is spatio-temporally chaotic.

It is noted that it is proved that the above equivalences also hold for sensitivity, multisensitivity, cofinite sensitivity, and Li-Yorke sensitivity (see [24, Corollary 0.1]). Also, we prove that if $p$ and $q$ are onto maps, then $p \circ q$ is spatio-temporally chaotic if and only if so is $q \circ p$. Moreover, we present two examples which show that for the above results it is necessary to assume that $p$ and $q$ are surjective maps.

\section{Preliminaries}

For the definition of Ruelle-Takens chaos, we refer the reader to [19]. For the notion of Kato chaos, we refer the reader to [7]. We know that topological mixing implies Kato's chaos [7, 27]. Moreover, Wu and Wang [27] proved that dense $\delta$-chaos, generic $\delta$-chaos, and Kato's chaos are equivalent.

Let $(Y, v)$ be a metric space and $\Gamma: Y \rightarrow Y$ be a continuous map. For any given $y_{1}, y_{2} \in Y$ and any given $s>0,\left(y_{1}, y_{2}\right)$ is said to be a Li-Yorke pair with modulus $s$ for $\Gamma$ if

$$
\liminf _{\mathfrak{m} \rightarrow \infty} v\left(\Gamma^{\mathrm{m}}\left(\mathrm{y}_{1}\right), \Gamma^{\mathrm{m}}\left(\mathrm{y}_{2}\right)\right)=0 \text { and } \limsup _{\mathrm{m} \rightarrow \infty} v\left(\Gamma^{\mathrm{m}}\left(\mathrm{y}_{1}\right), \Gamma^{\mathrm{m}}\left(\mathrm{y}_{2}\right)\right) \geqslant s .
$$

$\left(y_{1}, y_{2}\right)$ is a Li-Yorke pair for $\Gamma$ if it is a Li-Yorke pair with modulus $s$ for $\Gamma$ for some $s>0$. A continuous map $\Gamma: Y \rightarrow Y$ is said to be spatio-temporally chaotic if for any $y \in Y$ and any $\alpha>0$, there is some $x \in Y$ with $v(x, y)<\alpha$ such that $(x, y)$ is a Li-Yorke pair for $\Gamma$. For the concept of Li-Yorke sensitivity, we refer the reader to $[1,33]$. Clearly, by the definitions we can see that Li-Yorke sensitivity is different from spatio-temporal chaos, and that Li-Yorke sensitivity implies spatio-temporal chaos. 


\section{Main results}

Lemma 3.1. Let $\Gamma(\mathrm{s}, \mathrm{t})=(\mathrm{p}(\mathrm{t}), \mathrm{q}(\mathrm{s}))$ be a Cournot map over $\mathrm{G} \times \mathrm{H}$. Then the following hold:

1) if $\mathrm{q}$ is onto map and $\mathrm{p} \circ \mathrm{q}$ is spatio-temporally chaotic, then so is $\mathrm{q} \circ \mathrm{p}$;

2) if $\mathrm{p}$ is onto map and $\mathrm{q} \circ \mathrm{p}$ is spatio-temporally chaotic, then so is $\mathrm{p} \circ \mathrm{q}$.

Proof. Suppose that $p \circ q$ is spatio-temporally chaotic. Let $t \in H$ and $\mathrm{U}_{t} \subset \mathrm{H}$ be a neighborhood of $t \in \mathrm{H}$. Since $q$ is onto, one can pick $s \in q^{-1}(\{t\})$. Clearly, $q^{-1}\left(U_{t}\right)$ is a neighborhood of $s \in G$. By hypothesis and the definition, there is $s^{\prime} \in q^{-1}\left(u_{s}\right)$ such that

$$
\liminf _{j \rightarrow \infty}\left|(p \circ q)^{j}(s)-(p \circ q)^{j}\left(s^{\prime}\right)\right|=0 \quad \text { and } \quad \underset{j \rightarrow \infty}{\limsup }\left|(p \circ q)^{j}(s)-(p \circ q)^{j}\left(s^{\prime}\right)\right| \geqslant c
$$

for some $c>0$. As $p$ is uniformly continuous, there is $c^{\prime}>0$ such that if $\left|s_{1}-s_{2}\right|<c^{\prime}$ and $s_{1}, s_{2} \in H$, then $\left|p\left(s_{1}\right)-p\left(s_{2}\right)\right|<c$. So, we have that if

$$
\liminf _{j \rightarrow \infty}\left|(p \circ q)^{j}(s)-(p \circ q)^{j}\left(s^{\prime}\right)\right|=0,
$$

then

$$
\liminf _{j \rightarrow \infty}\left|q \circ(p \circ q)^{j}(s)-q \circ(p \circ q)^{j}\left(s^{\prime}\right)\right|=0,
$$

and that if

$$
\limsup _{j \rightarrow \infty}\left|(p \circ q)^{j}(s)-(p \circ q)^{j}\left(s^{\prime}\right)\right| \geqslant c,
$$

then

$$
\limsup _{j \rightarrow \infty}\left|q \circ(p \circ q)^{j}(s)-q \circ(p \circ q)^{j}\left(s^{\prime}\right)\right| \geqslant c^{\prime}
$$

In fact, if

$$
\limsup _{j \rightarrow \infty}\left|q \circ(p \circ q)^{j}(s)-q \circ(p \circ q)^{j}\left(s^{\prime}\right)\right|<c^{\prime},
$$

then

$$
\limsup _{j \rightarrow \infty}\left|p \circ\left(q \circ(p \circ q)^{j}\right)(s)-p \circ\left(q \circ(p \circ q)^{j}\right)\left(s^{\prime}\right)\right|<c,
$$

which implies

$$
\limsup _{j \rightarrow \infty}\left|(p \circ q)^{j}(s)-(p \circ q)^{j}\left(s^{\prime}\right)\right|<c .
$$

It is a contradiction. Thus, we have

$$
\liminf _{j \rightarrow \infty}\left|(q \circ p)^{j}(t)-(q \circ p)^{j}\left(t^{\prime}\right)\right|=0 \quad \text { and } \quad \limsup _{j \rightarrow \infty}\left|(q \circ p)^{j}(t)-(q \circ p)^{j}\left(t^{\prime}\right)\right| \geqslant c^{\prime},
$$

where $t^{\prime}=q\left(s^{\prime}\right) \in U_{t}$. By the definition, $q \circ p$ is spatio-temporally chaotic. Similarly, by hypothesis and the definition one can easily obtain that if $q \circ p$ is spatio-temporally chaotic, then $p \circ q$ is spatio-temporally chaotic.

Theorem 3.2. Let $\Gamma(\mathrm{s}, \mathrm{t})=(\mathrm{p}(\mathrm{t}), \mathrm{q}(\mathrm{s}))$ be a Cournot map over $\mathrm{G} \times \mathrm{H}$. If $\mathrm{p}$ and $\mathrm{q}$ are onto maps, then $\mathrm{p} \circ \mathrm{q}$ is spatio-temporally chaotic if and only if so is $\mathrm{q} \circ \mathrm{p}$.

Proof. By Lemma 3.1, the result of Theorem 3.2 is true.

We need the following lemmas to prove Theorem 3.6.

Lemma 3.3. If $\mathrm{p}: \mathrm{G} \rightarrow \mathrm{G}$ is a continuous selfmap over a compact metric space ( $\mathrm{G}, \mathrm{D})$, then $\mathrm{p}$ is spatio-temporally chaotic if and only if so is $\mathrm{p}^{2}$. 
Proof. By the definition, it is clear that if $p^{2}$ is spatio-temporally chaotic then so is $p$.

Now we assume that $p$ is spatio-temporally chaotic. By the definition, for any given $s \in G$ and any given $e>0$, there is $\mathrm{t} \in \mathrm{G}$ with $\mathrm{D}(\mathrm{s}, \mathrm{t})<\mathrm{e}$ satisfying that

$$
\liminf _{j \rightarrow \infty} D\left(p^{j}(s), p^{j}(t)\right)=0 \text { and } \limsup _{j \rightarrow \infty} D\left(p^{j}(s), p^{j}(t)\right) \geqslant c
$$

for some $c>0$. As $(G, D)$ is a compact metric space and $D$ is a continuous function, there is an increasing sequence $\left\{j_{i}\right\}_{i=1}^{\infty}$ of positive integers such that

$$
\lim _{i \rightarrow \infty} D\left(p^{2 j_{i}}(s), p^{2 j_{i}}(t)\right)=0 \quad \text { or } \quad \lim _{i \rightarrow \infty} D\left(p^{2 j_{i}+1}(s), p^{2 j_{i}+1}(t)\right)=0 .
$$

By the uniform continuity of $p$, if

$$
\lim _{i \rightarrow \infty} D\left(p^{2 j_{i}}(s), p^{2 j_{i}}(t)\right)=0 \quad \text { or } \quad \lim _{i \rightarrow \infty} D\left(p^{2 j_{i}+1}(s), p^{2 j_{i}+1}(t)\right)=0,
$$

then

$$
\lim _{i \rightarrow \infty} D\left(p^{2 j_{i}+2}(s), p^{2 j_{i}+2}(t)\right)=0
$$

This implies that

$$
\liminf _{j \rightarrow \infty} D\left(p^{2 j}(s), p^{2 j}(t)\right)=0 .
$$

By the uniform continuity of $p$, for the above $c>0$ there is $c^{\prime}>0$ with $c>c^{\prime}$ such that $D\left(s_{1}, s_{2}\right)<c^{\prime}$ with $s_{1}, s_{2} \in G$ implies $D\left(p^{j}\left(s_{1}\right), p^{j}\left(s_{1}\right)\right)<c^{\prime}$ for each $j \in\{1,2\}$. As $(G, D)$ is a compact metric space and $\mathrm{D}$ is a continuous function, there is an increasing sequence $\left\{k_{i}\right\}_{i=1}^{\infty}$ of positive integers such that

$$
\lim _{i \rightarrow \infty} D\left(p^{2 k_{i}}(s), p^{2 k_{i}}(t)\right) \geqslant c \quad \text { or } \quad \lim _{i \rightarrow \infty} D\left(p^{2 k_{i}+1}(s), p^{2 k_{i}+1}(t)\right) \geqslant c .
$$

So, if

$$
\lim _{i \rightarrow \infty} D\left(p^{2 k_{i}+1}(s), p^{2 k_{i}+1}(t)\right) \geqslant c,
$$

then

$$
\lim _{i \rightarrow \infty} D\left(p^{2 k_{i}}(s), p^{2 k_{i}}(t)\right) \geqslant c^{\prime}
$$

Consequently,

$$
\limsup _{j \rightarrow \infty} D\left(p^{2 j}(s), p^{2 j}(t)\right) \geqslant c^{\prime}
$$

Remark 3.4. By the proof of Lemma 3.3, one can similarly prove that if $p: G \rightarrow G$ is a continuous selfmap over a compact metric space $(G, D)$, then $p$ is spatio-temporally chaotic if and only if $p^{m}$ for some integer $m>1$ is spatio-temporally chaotic if and only if so is $p^{m}$ for any integer $m>1$.

Lemma 3.5. If $\mathrm{p}: \mathrm{G} \rightarrow \mathrm{G}$ (resp. $\mathrm{q}: \mathrm{H} \rightarrow \mathrm{H}$ ) is a continuous selfmap over a compact metric space $\left(\mathrm{G}, \mathrm{D}_{1}\right)$ (resp. $\left(\mathrm{H}, \mathrm{D}_{2}\right)$ ), then $\mathrm{p} \times \mathrm{q}$ is spatio-temporally chaotic if and only if the following conditions hold:

1) $\mathrm{p}$ is spatio-temporally chaotic;

2) q is spatio-temporally chaotic.

Proof. By the definition, it is easy to show that if $p$ (resp. $q$ ) is spatio-temporally chaotic, then so is $p \times q$.

Now we assume that $p \times q$ is spatio-temporally chaotic. Let $s \in G$ and $t \in H$, and let $A \subset G$ (resp.

$\mathrm{B} \subset \mathrm{H}$ ) be a neighborhood of $s($ resp. $t)$. Then, by the definition, there are $s^{\prime} \in A$ and $t^{\prime} \in B$ with

$$
\liminf _{j \rightarrow \infty} D\left((p \circ q)^{j}((s, t)),(p \circ q)^{j}\left(\left(s^{\prime}, t^{\prime}\right)\right)\right)=0 \quad \text { and } \quad \limsup _{j \rightarrow \infty} D\left((p \circ q)^{j}((s, t)),(p \circ q)^{j}\left(\left(s^{\prime}, t^{\prime}\right)\right)\right) \geqslant c
$$


for some $c>0$. As $(G \times H, D)$ is a compact metric space and $D$ is a continuous function, there is an increasing sequence $\left\{\dot{j}_{i}\right\}_{i=1}^{\infty}$ of positive integers such that

$$
\lim _{i \rightarrow \infty} D\left((p \circ q)^{j_{i}}((s, t)),(p \circ q)^{j_{i}}\left(\left(s^{\prime}, t^{\prime}\right)\right)\right)=0 .
$$

This implies that

$$
\liminf _{j \rightarrow \infty} D_{1}\left(p^{j}(s)-p^{j}\left(s^{\prime}\right)\right)=0 \quad \text { and } \quad \liminf _{j \rightarrow \infty} D_{2}\left(q^{j}(t)-q^{j}\left(t^{\prime}\right)\right)=0 .
$$

By the uniform continuity of $p$ and $q$, for the above $c>0$ there is $c^{\prime}>0$ with $c>c^{\prime}$ such that $D_{1}\left(s_{1}, s_{2}\right)<c^{\prime}$ with $s_{1}, s_{2} \in G$ implies $D_{1}\left(p\left(s_{1}\right), p\left(s_{1}\right)\right)<c^{\prime}$, and that $D_{1}\left(s_{1}, s_{2}\right)<c^{\prime}$ with $s_{1}, s_{2} \in G$ implies $D_{2}\left(q\left(t_{1}\right), q\left(t_{1}\right)\right)<c^{\prime}$. As $(G \times H, D)$ is a compact metric space and $D$ is a continuous function, there is an increasing sequence $\left\{k_{i}\right\}_{i=1}^{\infty}$ of positive integers such that

$$
\lim _{i \rightarrow \infty} D\left((p \times q)^{k_{i}}((s, t)),(p \times q)^{k_{i}}\left(\left(s^{\prime}, t^{\prime}\right)\right)\right) \geqslant c .
$$

Consequently, by the argument we get that

$$
\limsup _{j \rightarrow \infty} D_{1}\left(p^{j}(s), p^{j}\left(s^{\prime}\right)\right) \geqslant c^{\prime} \quad \text { and } \quad \limsup _{j \rightarrow \infty} D_{2}\left(q^{2 j}(t), q^{2 j}\left(t^{\prime}\right)\right) \geqslant c^{\prime} .
$$

Theorem 3.6. Let $\Gamma(\mathrm{s}, \mathrm{t})=(\mathrm{p}(\mathrm{t}), \mathrm{q}(\mathrm{s}))$ be a Cournot map over $\mathrm{G} \times \mathrm{H}$. If $\mathrm{p}$ and $\mathrm{q}$ are onto maps, then the following are equivalent:

(1) $\Gamma$ is spatio-temporally chaotic;

(2) $\left.\Gamma^{2}\right|_{\Lambda_{1}}$ is spatio-temporally chaotic;

(3) $\left.\Gamma^{2}\right|_{\Lambda_{2}}$ is spatio-temporally chaotic;

(4) $\left.\Gamma\right|_{\Lambda_{12}}$ is spatio-temporally chaotic.

Proof. Suppose that $\Gamma$ is spatio-temporally chaotic. By Lemma 3.3, $\Gamma^{2}$ is spatio-temporally chaotic. Since $\Gamma^{2}=(p \circ q) \times(q \circ p)$, by Lemma 3.5 we obtain that $p \circ q$ and $q \circ p$ are spatio-temporally chaotic.

Suppose that $p \circ q$ is spatio-temporally chaotic, and let $t \in H$ and $U_{t} \subset H$ be a neighborhood of $t \in H$. Since $q$ is onto, one can pick $s \in q^{-1}(\{t\})$. Clearly, $q^{-1}\left(U_{t}\right)$ is a neighborhood of $s \in G$. Then, $q^{-1}\left(U_{t}\right) \times U_{t}$ is a nonempty and open subset of $\Lambda_{2}$. As $p \circ q$ is spatio-temporally chaotic, by the definition, for the above $s \in \mathrm{q}^{-1}(\{\mathrm{t}\})$ and the neighborhood $\mathrm{q}^{-1}\left(\mathrm{U}_{\mathrm{t}}\right) \subset \mathrm{G}$ of $\mathrm{s}$, there is $\mathrm{s}^{\prime} \in \mathrm{q}^{-1}\left(\mathrm{U}_{\mathrm{t}}\right)$ with

$$
\liminf _{j \rightarrow \infty}\left|(p \circ q)^{j}(s)-(p \circ q)^{j}\left(s^{\prime}\right)\right|=0 \quad \text { and } \quad \limsup _{j \rightarrow \infty}\left|(p \circ q)^{j}(s)-(p \circ q)^{j}\left(s^{\prime}\right)\right| \geqslant c
$$

for some $c>0$. Clearly, there is an increasing sequence $\left\{\mathfrak{j}_{i}\right\}_{i=1}^{\infty}$ of positive integers such that

$$
\lim _{i \rightarrow \infty}\left|(p \circ q)^{j_{i}}(s)-(p \circ q)^{j_{i}}\left(s^{\prime}\right)\right|=0 .
$$

As q is uniformly continuous,

$$
\lim _{i \rightarrow \infty}\left|q \circ(p \circ q)^{j_{i}}(s)-q \circ(p \circ q)^{j_{i}}\left(s^{\prime}\right)\right|=0 .
$$

That is,

$$
\lim _{i \rightarrow \infty}\left|(q \circ p)^{j_{i}}(t)-(q \circ p)^{j_{i}}\left(t^{\prime}\right)\right|=0,
$$

where $t^{\prime}=q\left(s^{\prime}\right)$. Since

$$
\limsup _{j \rightarrow \infty}\left|(p \circ q)^{j}(s)-(p \circ q)^{j}\left(s^{\prime}\right)\right| \geqslant c,
$$


there is an increasing sequence $\left\{k_{i}\right\}_{i=1}^{\infty}$ of positive integers such that

$$
\lim _{i \rightarrow \infty}\left|(p \circ q)^{k_{i}}(s)-(p \circ q)^{k_{i}}\left(s^{\prime}\right)\right| \geqslant c .
$$

By the uniform continuity of $p$, for the above $c>0$ there is $c^{\prime}>0$ with $c>c^{\prime}$ such that $\left|s_{1}-s_{2}\right|<c^{\prime}$ with $s_{1}, s_{2} \in H$ implies $\left|p\left(s_{1}\right)-p\left(s_{1}\right)\right|<c^{\prime}$. So,

$$
\lim _{i \rightarrow \infty}\left|q \circ(p \circ q)^{k_{i}}(s)-q \circ(p \circ q)^{k_{i}}\left(s^{\prime}\right)\right| \geqslant c^{\prime} .
$$

That is,

$$
\lim _{i \rightarrow \infty}\left|(q \circ p)^{k_{i}}(t)-(q \circ p)^{k_{i}}\left(t^{\prime}\right)\right| \geqslant c^{\prime} .
$$

Consequently, by the argument we get that

$$
\liminf _{j \rightarrow \infty} D\left(\Gamma^{2 j}((s, t)), \Gamma^{2 j}\left(\left(s^{\prime}, t^{\prime}\right)\right)\right)=0 \quad \text { and } \quad \limsup _{j \rightarrow \infty} D\left(\Gamma^{2 j}((s, t)), \Gamma^{2 j}\left(\left(s^{\prime}, t^{\prime}\right)\right)\right) \geqslant c^{\prime} .
$$

By the definition, $\left.\Gamma^{2}\right|_{\Lambda_{2}}$ is spatio-temporally chaotic. Similarly, we can show that if $q \circ p$ is spatiotemporally chaotic, then so is $\left.\Gamma^{2}\right|_{\Lambda_{1}}$.

Assume that $\left.\Gamma^{2}\right|_{\Lambda_{1}}$ (resp. $\left.\Gamma^{2}\right|_{\Lambda_{2}}$ ) is spatio-temporally chaotic. By the definition, $\Gamma^{2}$ is spatio-temporally chaotic. By Lemma 3.3, $\Gamma$ is spatio-temporally chaotic. As $\Gamma\left(\Lambda_{12}\right) \subset \Lambda_{12}, \Gamma_{\Lambda_{12}}$ is spatio-temporally chaotic.

Suppose that $\Gamma_{\Lambda_{12}}$ is spatio-temporally chaotic. By the definition, $\Gamma$ is spatio-temporally chaotic. So, by the above argument, $\left.\Gamma^{2}\right|_{\Lambda_{1}}\left(\right.$ resp. $\left.\Gamma^{2}\right|_{\Lambda_{2}}$ ) is spatio-temporally chaotic.

Remark 3.7. By Remark 3.4, Lemma 3.5, and Theorems 3.2 and 3.6, one can similarly prove that for a Cournot map $\Gamma(s, t)=(p(t), q(s))$ over $G \times H$, if $p$ and $q$ are onto maps, then the following are equivalent:

(1) $\Gamma$ is spatio-temporally chaotic;

(2) $\left.\Gamma^{2}\right|_{\Lambda_{1}}$ is spatio-temporally chaotic;

(3) $\left.\Gamma^{2}\right|_{\Lambda_{2}}$ is spatio-temporally chaotic;

(4) $\left.\Gamma\right|_{\Lambda_{12}}$ is spatio-temporally chaotic;

(5) $\Gamma^{\mathrm{m}}$ is spatio-temporally chaotic for some integer $\mathrm{m}>1$;

(6) $\left.\Gamma^{2 m}\right|_{\Lambda_{1}}$ is spatio-temporally chaotic for some integer $m>1$;

(7) $\left.\Gamma^{2 m}\right|_{\Lambda_{2}}$ is spatio-temporally chaotic for some integer $m>1$;

(8) $\Gamma^{\mathrm{m}} \mid \Lambda_{12}$ is spatio-temporally chaotic for some integer $\mathrm{m}>1$;

(9) $\Gamma^{\mathrm{m}}$ is spatio-temporally chaotic;

(10) $\left.\Gamma^{2 m}\right|_{\Lambda_{1}}$ is spatio-temporally chaotic for any integer $m>1$;

(11) $\left.\Gamma^{2 m}\right|_{\Lambda_{2}}$ is spatio-temporally chaotic for any integer $m>1$;

(12) $\left.\Gamma^{\mathrm{m}}\right|_{\Lambda_{12}}$ is spatio-temporally chaotic for any integer $m>1$.

Example 3.8. Let $X=[0,1]$ and $Y=[0,2]$. Define $p: X \rightarrow Y$ and $q: Y \rightarrow X$ by $p(x)=1-|1-2 x|$ for any $x \in X, q(y)=1-|1-2 y|$ for any $y \in X$, and $q(y)=1-|1-2(y-1)|$ for any $y \in Y$ with $y \notin X$. Then, the following hold:

(1) $q \circ p$ is spatio-temporally chaotic;

(2) $\mathrm{p} \circ \mathrm{q}$ is spatio-temporally chaotic.

Proof. As $\left.(p \circ q)\right|_{X}$ is the tent map which is topologically exact, the map $\left.(p \circ q)\right|_{X}$ satisfies the condition of Theorem 1 in [9] which implies $(p \circ q)$ satisfies the condition of Theorem 1 in [9]. By the definition and Theorem 1 in [9], $\mathrm{p} \circ \mathrm{q}$ is spatio-temporally chaotic. As $\mathrm{q}$ is surjective, by Lemma 3.1, $\mathrm{q} \circ \mathrm{p}$ is spatiotemporally chaotic. 
Example 3.9. Let $X=[0,1]$ and $Y=[0,2]$. Define $p: X \rightarrow Y$ and $q: Y \rightarrow X$ by $p(x)=1-|1-2 x|$ for any $x \in X, q(y)=1-|1-2 y|$ for any $y \in X$, and $q(y)=0$ for any $y \in Y$ with $y \notin X$ (see [13]). Then, the following hold:

(1) $q \circ p$ is spatio-temporally chaotic;

(2) $\mathrm{p} \circ \mathrm{q}$ is not spatio-temporally chaotic.

Proof. As $q \circ p$ is the tent map, by the proof of Example 3.8 we know that $q \circ p$ is spatio-temporally chaotic. By definition one can easily see that $\mathrm{p} \circ \mathrm{q}$ is not spatio-temporally chaotic.

Remark 3.10. Example 3.9 shows that for the main results in this paper, it is necessary to assume that $p$ and $\mathrm{q}$ are onto maps.

\section{Acknowledgment}

The authors are very grateful to the referees for their careful reading, comments, and suggestions, which help us to improve this paper.

This research was supported by the Project of Enhancing School With Innovation of Guangdong Ocean University (Grant NO. GDOU2016050207), the Key Scientific and Technological Research Project of Science and Technology Department of Zhanjiang City (Grant 2010C3112005), the National Natural Science Foundation of China(11501391), the Opening Project of Artificial Intelligence Key Laboratory of Sichuan Province (2015RZJ01), and the Opening Project of Bridge Non-destruction Detecting and Engineering Computing Key Laboratory of Sichuan Province (2014QZJ02).

\section{References}

[1] E. Akin, S. Kolyada, Li-Yorke sensitivity, Nonlinearity, 16 (2003), 1421-1433. 1, 2

[2] G. I. Bischi, C. Mammana, L. Gardini, Multistability and cyclic attractors in duopoly games, Chaos Solitons Fractals, 11 (2000), 543-564. 1

[3] J. S. Cánovas, Chaos in duopoly games, Nonlinear Stud., 7 (2000), 97-104. 1

[4] J. S. Cánovas, M. Ruíz Marín, Chaos on MPE-sets of duopoly games, Dedicated to our teacher, mentor and friend, Nobel laureate, Ilya Prigogine, Chaos Solitons Fractals, 19 (2004), 179-183. 1

[5] J. S. Cánovas Peña, G. Soler López, M. Ruiz Marín, Distributional chaos of Cournot maps, Adv. Nonlinear Stud., 1 (2001), 79-87.

[6] R. A. Dana, L. Montrucchio, Dynamic complexity in duopoly games, J. Econom. Theory, 44 (1986), 40-56. 1

[7] H. Kato, Everywhere chaotic homeomorphisms on manifolds and k-dimensional Menger manifolds, Topology Appl., 72 (1996), 1-17. 2

[8] M. Kopel, Simple and complex adjustment dynamics in Cournot duopoly models, Complex dynamics in economic and social systems, Umeå, (1995), Chaos Solitons Fractals, 7 (1996), 2031-2048. 1

[9] R.-S. Li, A note on distributional chaos of periodically adsorbing systems, (Chinese) J. Systems Sci. Math. Sci., 32 (2012), 237-243. 3

[10] R.-S. Li, A note on stronger forms of sensitivity for dynamical systems, Chaos Solitons Fractals, 45 (2012), 753-758. 1

[11] R.-S. Li, H.-Q. Wang, Y. Zhao, Kato's chaos in duopoly games, Chaos Solitons Fractals, 84 (2016), 69-72. 1

[12] T. Y. Li, J. A. Yorke, Period three implies chaos, Amer. Math. Monthly, 82 (1975), 985-992. 1

[13] T.-X. Lu, P.-Y. Zhu, Further discussion on chaos in duopoly games, Chaos Solitons Fractals, 52 (2013), 45-48. 1, 3.9

[14] P. Oprocha, P. Wilczyński, Shift spaces and distributional chaos, Chaos Solitons Fractals, 31 (2007), 347-355. 1

[15] R. Pikula, On some notions of chaos in dimension zero, Colloq. Math., 107 (2007), 167-177. 1

[16] T. Puu, Chaos in duopoly pricing, Chaos Solitons Fractals, 1 (1991), 573-81. 1

[17] T. Puu, I. Sushko, Oligopoly and complex dynamics, Springer, New York, (2002). 1

[18] D. Rand, Exotic phenomena in games and duopoly models, J. Math. Econom., 5 (1978), 173-84. 1

[19] D. Ruelle, F. Takens, On the nature of turbulence, Comm. Math. Phys., 20 (1971), 167-92. 2

[20] B. Schweizer, J. Smítal, Measures of chaos and a spectral decomposition of dynamical systems on the interval, Trans. Amer. Math. Soc., 344 (1994), 737-754. 1

[21] J. Smítal, M. Štefánková, Distributional chaos for triangular maps, Chaos Solitons Fractals, 21 (2004), 1125-1128. 1

[22] L.-D. Wang, G.-F. Huang, S.-M. Huan, Distributional chaos in a sequence, Nonlinear Anal., 67 (2007), 2131-2136. 1

[23] X.-X. Wu, Chaos of transformations induced onto the space of probability measures, Internat. J. Bifur. Chaos Appl. Sci. Engrg., 26 (2016), 12 pages. 1 
[24] X.-X. Wu, A remark on topological sequence entropy, Internat. J. Bifur. Chaos Appl. Sci. Engrg., 27 (2017), 7 pages. 1, 1

[25] X.-X. Wu, G.-R. Chen, Sensitivity and transitivity of fuzzified dynamical systems, Inform. Sci., 396 (2017), 14-23. 1

[26] X.-X. Wu, P. Oprocha, G.-R. Chen, On various definitions of shadowing with average error in tracing, Nonlinearity, 29 (2016), 1942-1972. 1

[27] X.-X. Wu, J.-J. Wang, A remark on accessibility, Chaos Solitons Fractals, 91 (2016), 115-117. 2

[28] X.-X. Wu, X. Wang, On the iteration properties of large deviations theorem, Internat. J. Bifur. Chaos Appl. Sci. Engrg., 26 (2016), 6 pages. 1

[29] X.-X. Wu, J.-J. Wang, G.-R. Chen, $\mathscr{F}$-sensitivity and multi-sensitivity of hyperspatial dynamical systems, J. Math. Anal. Appl., 429 (2015), 16-26.

[30] X.-X. Wu, X. Wang, G.-R. Chen, On the large deviations of weaker types, Internat. J. Bifur. Chaos Appl. Sci. Engrg., 27 (2017), 12 pages.

[31] X.-X. Wu, L.-D. Wang, G.-R. Chen, Weighted backward shift operators with invariant distributionally scrambled subsets, Ann. Funct. Anal., 8 (2017), 199-210. 1

[32] X.-X. Wu, L.-D. Wang, J.-H. Liang, The Chain Properties and Average Shadowing Property of Iterated Function Systems, Qual. Theory Dyn. Syst., 2016 (2016), 9 pages.

[33] X.-X. Wu, L.-D. Wang, J.-H. Liang, The chain properties and Li-Yorke sensitivity of zadehs extension on the space of upper semi-continuous fuzzy sets, Iran. J. Fuzzy Syst., (Accepted). 1, 2 